\title{
Analysis of Differences in Gene Expression Associated with Variation in Biomass Composition in Sugarcane ${ }^{+}$
}

\author{
Virginie Perlo *, Agnelo Furtado, Frikkie Botha and Robert Henry \\ Queensland Alliance for Agriculture and Food Innovation, University of Queensland, Brisbane, QLD 4072, \\ Australia; a.furtado@uq.edu.au (A.F.); f.botha@uq.edu.au (F.B.); robert.henry@uq.edu.au (R.H.) \\ * Correspondence: v.perlo@uq.edu.au \\ + Presented at the third International Tropical Agriculture Conference (TROPAG 2019), Brisbane, Australia, \\ 11-13 November 2019.
}

Published: 7 April 2020

\begin{abstract}
Sugarcane has a high potential to support second-generation ethanol production and environmentally friendly by-products for use in chemical, pharmaceutical, medical, cosmetic and food industries. A crucial challenge for a long-term economic viability is to optimise the crop for production of a biomass composition that will ensure maximum economic benefit. Transcriptome data analysis provides a relevant explanation of phenotypic variances and gives a more accurate prediction of phenotypes than genomic information. This multi-omic approach, with an integrated transcriptomics and metabolomics analysis may reveal details of biological mechanisms and pathways. A global view of transcriptional regulation and the identification differentially expressed genes (DEGs) and metabolites may help the feasibility of tailoring engineering targeted biosynthetic pathways to improve the production of these bio-products from sugarcane. We propose a profiling analysis workflow (pipeline) to generate empirical correlations between gene expression, metabolites, proteins and phenotypic traits and pathway analysis, with a highlight focus on data visualisation. This study of genetic variation in gene expression and correlations with metabolic and protein phenotype relies on high-throughput methodology, measurement and analysis of 360 samples, 24 commercial sugarcane cultivars with different phenotypic characteristics at 5 different development stages with 3 replicates.
\end{abstract}

Keywords: transcriptome; metabolome; sugarcane 